\title{
Can beauty be ignored? Effects of facial attractiveness on covert attention
}

\author{
JIE SUI \\ University of Hull, Hull, England \\ and Northeast Normal University, Changchun, Jilin, China \\ AND \\ Chang Hong Liu \\ University of Hull, Hull, England
}

\begin{abstract}
Facial beauty has important social and biological implications. Research has shown that people tend to look longer at attractive than at unattractive faces. However, little is known about whether an attractive face presented outside foveal vision can capture attention. The effect of facial attractiveness on covert attention was investigated in a spatial cuing task. Participants were asked to judge the orientation of a cued target presented to the left or right visual field while ignoring a task-irrelevant face image flashed in the opposite field. The presentation of attractive faces significantly lengthened task performance. The results suggest that facial beauty automatically competes with an ongoing cognitive task for spatial attention.
\end{abstract}

There is now substantial evidence that faces capture attention more than do most common objects (e.g., Ro, Russell, \& Lavie, 2001). Moreover, certain facial information, such as expressions of anger and fear, evokes attention more easily than do neutral faces. Attention to such information is often rapid, unconscious, and mandatory (see Palermo \& Rhodes, 2007, for a review). In the present study, we examine whether the same characteristics are found in attention for facial beauty. This should offer important insights about how attentional systems prioritize and select meaningful biological information. The impact of beauty on social behavior has been well documented in the literature (Langlois et al., 2000). However, unlike attention to emotions, attention to facial attractiveness has been studied little.

Olson and Marshuetz (2005) have shown that facial beauty is appraised even when face images are presented for less than $20 \mathrm{msec}$ and followed by backward masking. Their study suggests that facial attractiveness can be detected rapidly from transient and degraded visual information. Other studies have shown that participants tend to look longer at attractive than at unattractive faces (Aharon et al., 2001) or take longer to decide on the attractiveness of attractive than on that of unattractive faces (Kranz \& Ishai, 2006). Recent literature also has suggested that neural responses to facial beauty are engaged automatically, because they can be measured even when participants are performing a task unrelated to the explicit task of judging facial attractiveness (Aharon et al., 2001; O'Doherty et al., 2003; Winston, O'Doherty, Kilner, Perrett, \& Dolan, 2007). However, because attention in these studies was focused directly on the location where face images were presented, it is not clear whether appraisal for attractiveness can be achieved if spatial attention has already been directed elsewhere. Is the appraisal for beauty mandatory in such a way that attractive faces can compete automatically with an ongoing task for attentional resources? Also, because faces were presented at fixation in these studies, it is not clear whether facial attractiveness can be detected outside the foveal vision.

To find answers to these questions, we employed a spatial endogenous cuing task in which participants were asked to determine whether a laterally presented target letter was upright or inverted and to ignore a face (which they were told was task irrelevant) flashed briefly on the opposite side of the display. The likely position of the forthcoming target was indicated by a central cue, which was used to induce covert attention (i.e., visual attention without orienting eye movements) to the cued location. We examined the presence of attractive faces on the task performance. We hypothesized that, relative to the presence of an unattractive face, the presence of an attractive face would create a stronger interference with the task because attractive faces may automatically pull the attention away from the target.

To assess the effect of facial attractiveness on covert orienting, the face image and the target in each trial were presented simultaneously. Because the duration of a single eye fixation usually exceeds $200 \mathrm{msec}$ (Rayner, 1983), the target and the face in this study were shown for no longer than $200 \mathrm{msec}$, to suppress saccadic orienting to

J. Sui, jie.sui@hull.ac.uk 
the stimuli. To make the task more difficult, the presentation time for these was reduced further from $200 \mathrm{msec}$ in Experiment 1 to $100 \mathrm{msec}$ in Experiment 2. If attractive faces capture spatial attention automatically, the effects should be relatively independent of task difficulty. Finally, we conducted Experiment 3 to replicate our main findings and to determine the role of eye movements.

\section{METHOD}

\section{Participants}

A total of 142 undergraduate and graduate students from the University of Hull participated in this study. Experiment 1 had 40 participants (30 females, 10 males; ages 18-40 years; mean age $23 \pm 6.29$ years). Experiment 2 had 43 participants ( 27 females, 16 males; ages $18-45$ years, mean age $20 \pm 3.92$ years). Experiment 3 had 59 participants ( 35 females, 24 males; ages 19-34 years; mean age $21 \pm 2.11$ years). All participants had normal or corrected-tonormal vision.

\section{Stimuli}

The face database was obtained from the University of St. Andrews. It contains 702 frontal-view Caucasian faces whose external features (hair and clothing) were removed. All faces in the database were prerated by 19 raters (ages 18-29 years; 12 females) for attractiveness on a 7-point scale. To contrast the effect of attractiveness, only the 82 most attractive and 82 least attractive faces were used. The mean ratings for the two groups of faces were 4.11 and 2.23 , respectively. These were significantly different from each other $(p<.001)$. Both the attractive and the unattractive face groups contained equal numbers of males and females. Four of these faces were reserved as the practice stimuli. The face size was normalized to 400 pixels from ear to ear, which subtended $16.6^{\circ}$ of visual angle. Because face identification is highly sensitive to image contrast in peripheral presentation (Mäkelä, Näsänen, Rovamo, \& Melmoth, 2001; Melmoth, Kukkonen, Mäkelä, \& Rovamo, 2000), the luminance and contrast of the images were scaled to their means, so that these low-level image properties could contribute little to any behavioral difference.

The cuing displays consisted of a central fixation point, a cue, and two $3.8^{\circ} \times 3.8^{\circ}$ white boxes on a neutral gray background (see Figure 1). The distance between the center of the display and the outer edge of each box measured $5.5^{\circ}$ of visual angle. The cue was a $1.5^{\circ}$ white arrow. The target display consisted of a target letter " $\mathrm{T}$ " embedded in the center of an array of eight distractor crosses (see Figure 1). This configuration was the same from trial to trial. The size of the target and distractors was $1.2^{\circ} \times 1.2^{\circ}$. The letter " $\mathrm{T}$ " was shown either upright or inverted. E-Prime 1.1 was used to control the flow of the experiment and to collect response data.

\section{Procedure}

Participants were tested individually. An adjustable headrest was used to fix the participant's viewing position, which was set $60 \mathrm{~cm}$ away from the computer monitor. The procedure for each trial of the experiments is illustrated in Figure 1. Each trial began with a central fixation cross and two peripheral boxes. The fixation cross was shown for $500 \mathrm{msec}$. It was then replaced by a $200-\mathrm{msec}$ central cue, which pointed randomly to the right or left box. In $80 \%$ of trials (valid trials), the cue indicated the target location. In the remaining $20 \%$ (invalid trials), the cue pointed to the wrong location. The interstimulus interval before the target presentation was 50 or $150 \mathrm{msec}$. The stimulus onset asynchrony (SOA), or the interval between the onset of the cue and that of the target, was thus 250 or $350 \mathrm{msec}$. As has been reported in the literature, SOAs around this range consistently produce a relatively strong attention-orienting effect, in which valid cues create faster responses to a target than do invalid cues (Funes, Lupiáñez, \& Milliken, 2007). We used the two SOAs to determine the temporal window within which the effect of

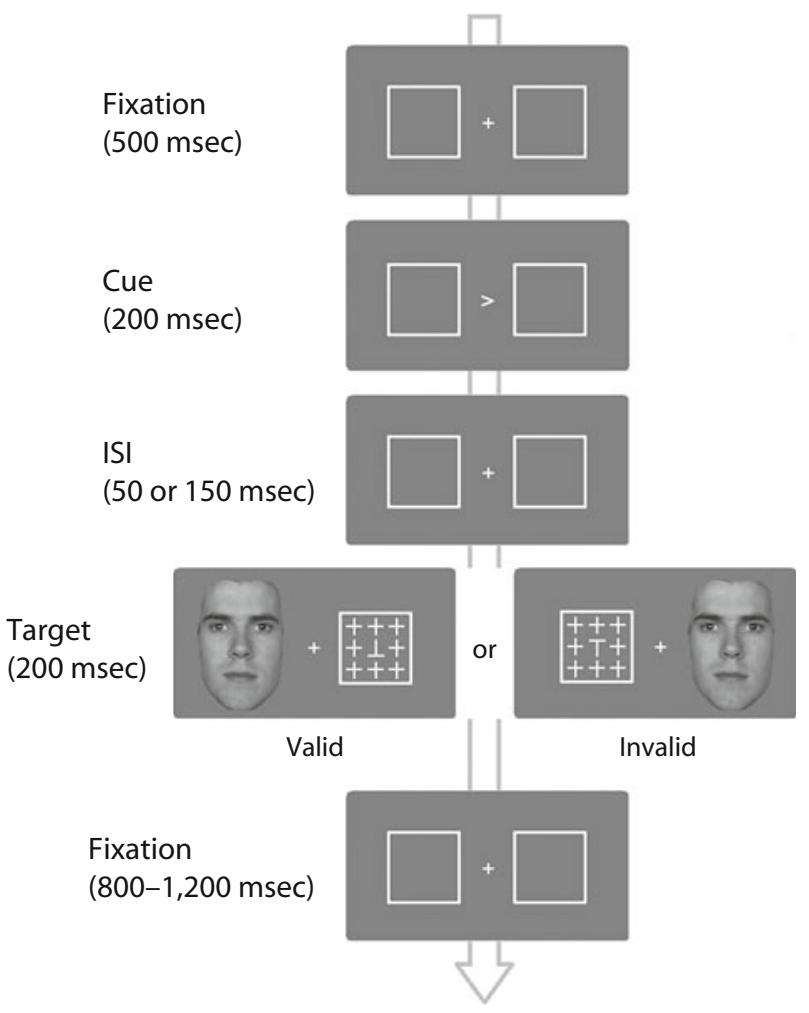

Figure 1. Illustration of the procedure used in the study. The target duration in Experiment 2 was changed from 200 to 100 msec.

facial attractiveness on attentional orienting is maximal. In the experimental conditions, an attractive or unattractive face was shown simultaneously with the target for $200 \mathrm{msec}$ (Experiments 1 and 3) or $100 \mathrm{msec}$ (Experiment 2). Experiments 1 and 2 were identical, except for the different target and face durations. In the baseline condition, the target and peripheral box were shown without a face for the same duration. We used this condition to evaluate the difference between the results of face-present and face-absent trials. The next frame showed the fixation point and peripheral boxes for a time ranging from 800 to $1,200 \mathrm{msec}$, to prevent easy predictions about the onset of the next trial. Participants were expected to respond within this time frame, and the next trial started regardless of whether a response was recorded. Participants were told to ignore the faces while judging whether the target letter "T," presented on the opposite side, was upright or inverted. They were instructed to respond as quickly and accurately as possible by pressing one of the two specified keys on the keyboard. Participants were also reminded to maintain central fixation throughout the trials.

In Experiments 1 and 2, a total of 80 practice trials were run before the 720 experimental trials. Each of the 12 conditions ( 3 irrelevant stimuli $\times 2$ visual fields $\times 2$ SOAs) in the experimental trials had 48 valid and 12 invalid trials. Participants were given short breaks after every 240 trials. Experiment 3 was identical to Experiment 1, except that the long SOA (350-msec) condition was excluded. This change was made because neither Experiment 1 nor Experiment 2 showed any effect of facial attractiveness at this SOA.

Because the interval between the cue and target onset was $250 \mathrm{msec}$ or longer in this study, the results in these experiments could be affected by stimulus-driven eye movements, even though the participants were told to maintain fixation. To test this possibility, in Experiment 3 we examined potential contributions of eye movements by recording electrooculograms (EOGs) of 5 participants (see Hawkins et al., 1990, for more details about this method). The EOGs were re- 


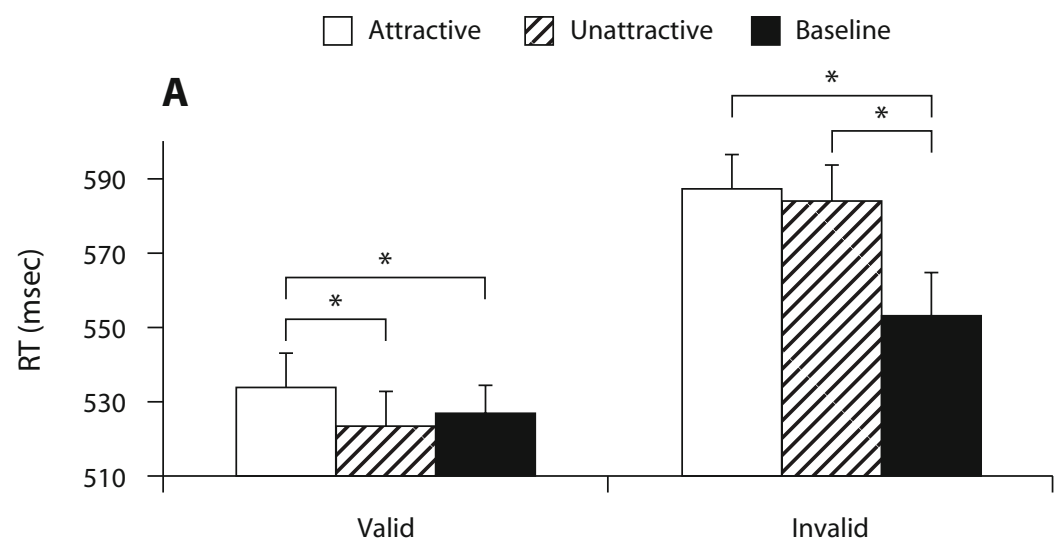

B

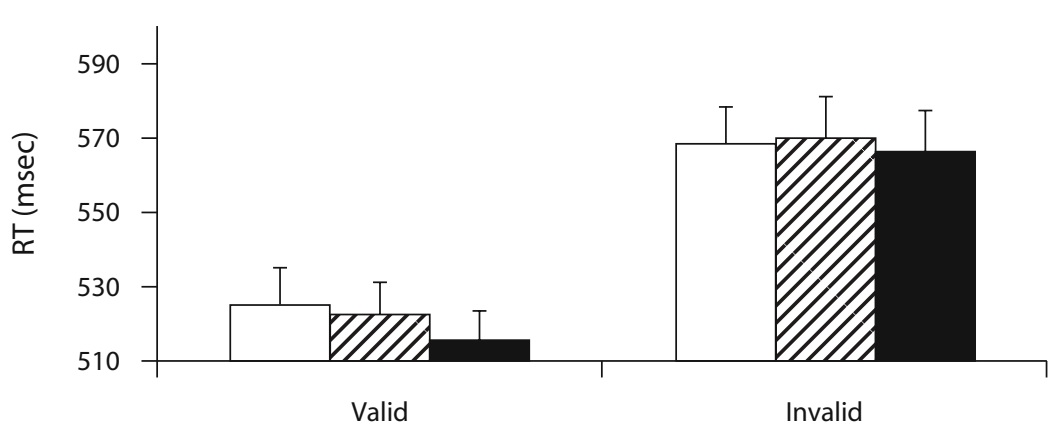

Figure 2. Mean reaction time (RT) in Experiment 1 as a function of irrelevant stimuli, stimulus onset asynchrony (SOA), and cue validity. Error bars represent standard errors. (A) $\mathrm{SOA}=250 \mathrm{msec}$. (B) $\mathrm{SOA}=350 \mathrm{msec}$.

corded with two pairs of electrodes, with a passband of $0.1-100 \mathrm{~Hz}$, and were digitized at a sampling rate of $250 \mathrm{~Hz}$. The horizontal EOG was recorded from electrodes placed about $1.5 \mathrm{~cm}$ lateral to the left and right external canthi, and the vertical EOG was recorded with electrodes located above and under the left eye. Eye movement on a given trial was defined by deflections exceeding $\pm 50 \mu \mathrm{V}$.

\section{RESULTS}

The data were analyzed using repeated measures ANOVAs. The four variables were irrelevant stimuli (attractive, unattractive, baseline), cue validity (valid vs. invalid), visual field (right vs. left), and SOA (250 vs. $350 \mathrm{msec}$ ). The overall accuracy results in all experiments were high $(87 \%-95 \%)$. Because no significant difference was found between the accuracy results for attractive and unattractive faces, we focus here mainly on the reaction time (RT) data. Analyses of RT were based on the data for the correct responses only. RT outliers were defined as 3 $S D$ s outside of the mean. We did not detect any outlier, using this criterion.

\section{Experiment 1}

RT results from Experiment 1 are shown in Figure 2. Initial analysis showed a significant main effect of visual field $[F(1,39)=11.65, p<.005]$, where responses were faster when the target was presented in the left than in the right visual field. There was also a main effect of irrelevant stimuli $[F(2,78)=9.10, p<.005]$, in instances where RT was significantly longer for attractive and unattractive faces than in the baseline condition $(p \mathrm{~s}<.01)$. However, since there were no significant interactions involving visual field and irrelevant stimuli (all $p$ s $>.32$ ), the data from the two visual fields were combined in the subsequent analyses. This showed a significant three-way interaction of irrelevant stimuli $\times$ cue validity $\times$ SOA $[F(2,78)=6.04, p<.005]$.

To identify the source of this interaction, we conducted two separate simple main effects analyses for the two SOA conditions, which revealed that the effect of irrelevant stimuli occurred only at the shorter SOA $(250 \mathrm{msec})$. As expected, valid cues produced faster RTs than did invalid cues $[F(1,39)=34.39, p<.0001]$. We also found a significant interaction between irrelevant stimuli and cue validity $[F(2,78)=7.54, p<.001]$. Separate ANOVAs were conducted for the invalid and valid cue conditions. A significant main effect of irrelevant stimuli was found in the valid cue condition $[F(2,78)=8.18, p<.001]$. Consistent with our hypothesis, attractive faces delayed RTs more than did unattractive faces or the baseline condition $[t \mathrm{~s}(39)=4.21$ and $2.75, p \mathrm{~s}<.001$ and .01 , respectively $]$. No difference was found between RTs in the unattractive and baseline conditions $[t(39)=-1.10, p=.28]$. The effect implies that facial beauty can trigger processes that 

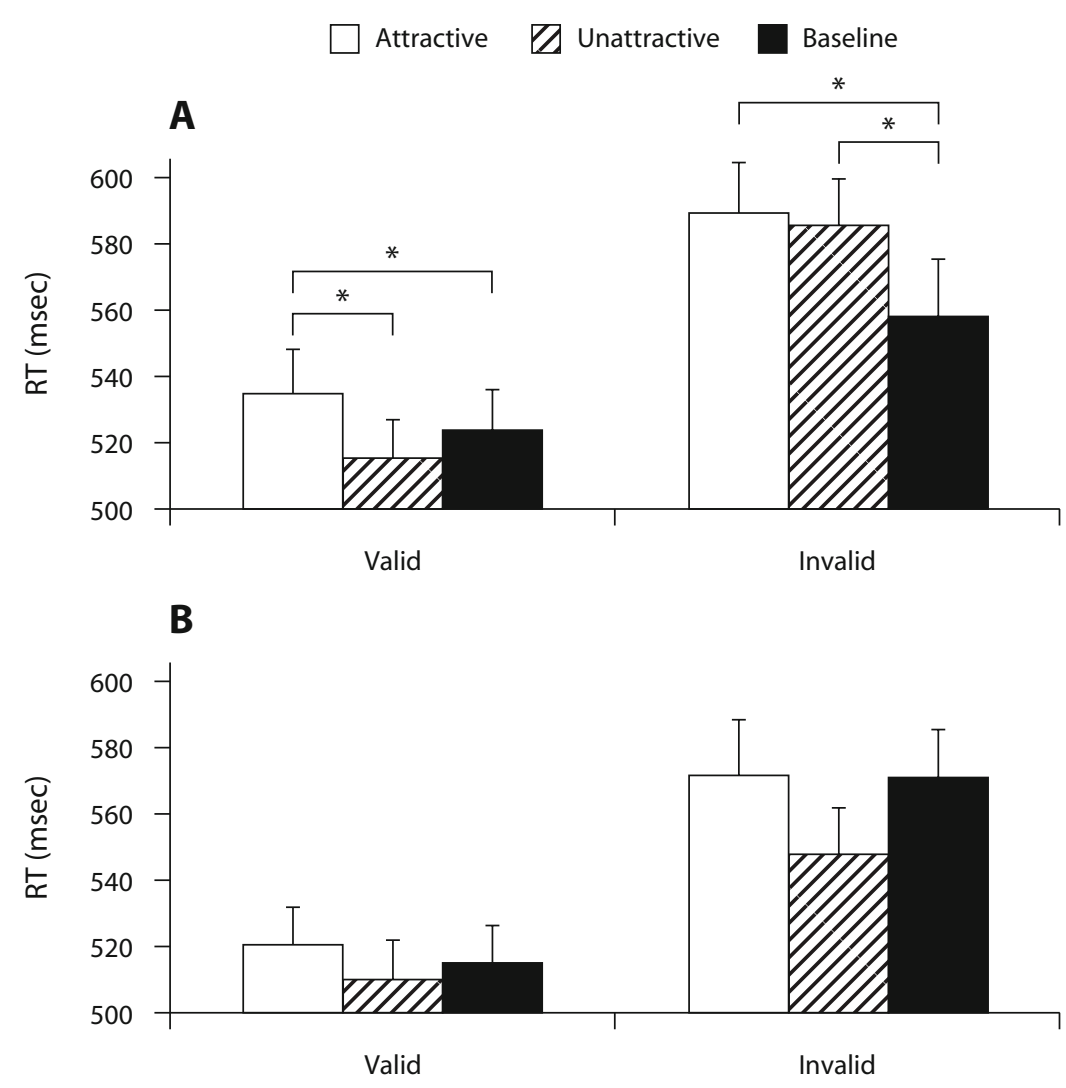

Figure 3. Mean reaction time (RT) in Experiment 2 as a function of irrelevant stimuli and cue validity $(\mathrm{SOA}=\mathbf{2 5 0} \mathbf{m s e c})$. Error bars represent standard errors. (A) Face on the left and target in the right visual field. (B) Face on the right and target in the left visual field.

compete with an ongoing cognitive task for attentional resources. In the invalid cue condition, on the other hand, RTs for attractive and unattractive faces were comparable $[t(42)=0.76, p=.45]$, although both were slower than RTs in the baseline condition $[t \mathrm{~s}(42)=3.43$ and 3.19 , $p$ s $<.001$ and .005 , respectively].

\section{Experiment 2}

Experiment 1 showed that attractive faces affect spatial attention more strongly than unattractive faces did in the valid cue condition. Experiment 2 further investigated whether the effect could be replicated when the duration of target and face stimuli was reduced from 200 to $100 \mathrm{msec}$.

Analysis of the RT data showed that the main effect of SOA was significant $[F(1,42)=5.71, p<.02]$ : Responses were faster for the long SOA than for the short SOA. There was also a main effect of irrelevant stimuli $[F(2,84)=4.83$, $p<.01]$ : Only attractive faces lengthened RT relative to the baseline condition $(p<.005)$. An ANOVA showed a significant four-way interaction $[F(2,84)=6.82, p<.005]$. Separate ANOVAs for the two visual fields revealed that the effect of irrelevant stimuli was present when faces were presented in the left visual field $[F(2,84)=5.26, p<.01]$, but not when they were presented in the right visual field $(p=.47)$. Results from presentations in the left visual field are shown in Figure 3A. For comparison, results from presentations in the right visual field are shown in Figure 3B. Only short SOA results from this experiment are presented, because our subsequent analysis did not show effects of facial attractiveness in the long SOA condition.

All interactions involving irrelevant stimuli for the right visual field (irrelevant stimuli $\times$ cue validity, irrelevant stimuli $\times$ SOA, and irrelevant stimuli $\times$ cue validity $\times$ SOA) were also not significant ( $p \mathrm{~s}>.1)$. The results for visual field in this experiment were thus different from those in Experiment 1, where the effect of facial attractiveness was present in both visual fields. Our main statistical analysis was therefore conducted on the condition in which the face stimuli were presented in the left visual field. Results from this condition revealed a significant three-way interaction of irrelevant stimuli $\times$ cue validity $\times \operatorname{SOA}[F(2,84)=6.15, p<.005]$. Separate ANOVAs were conducted for the two SOA conditions. As in Experiment 1, the results for the long SOA showed no significant main effect of irrelevant stimuli or interaction between this and cue validity ( $p \mathrm{~s}>.19$ ), and only the short SOA produced a significant interaction between irrelevant stimuli and cue validity $[F(2,84)=6.16, p<.005]$. Also as in Experiment 1, the RT was significantly slower when an attractive face was shown with the target than when an unattractive face $[t(42)=3.74, p<.001]$ or baseline $[t(42)=$ 


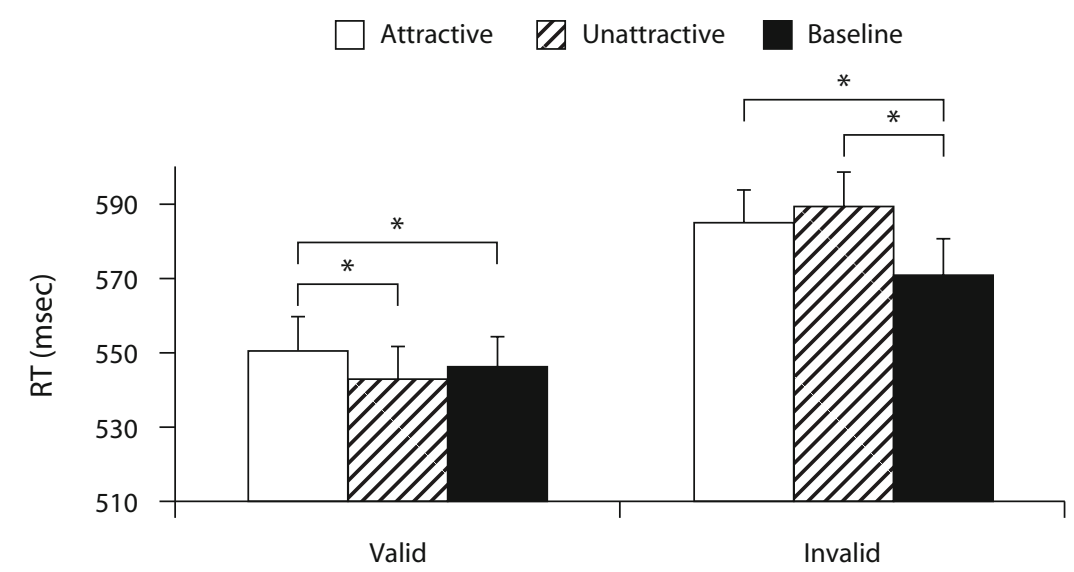

Figure 4. Mean reaction time (RT) in Experiment 3 as a function of irrelevant stimuli and cue validity $(\mathrm{SOA}=\mathbf{2 5 0} \mathrm{msec})$. Error bars represent standard errors.

2.13, $p<.04$ ] was shown with the target. Again the effect was observed only when the cue was valid. When the cue was invalid, RTs for the attractive and unattractive face conditions did not differ $(p=.57)$, although both attractive and unattractive face conditions delayed response to the target relative to the baseline condition $[t \mathrm{~s}(42)=3.74$ and 2.20, $p$ s $<.001$ and .04 , respectively].

Experiment 2 showed that the facial attractiveness effects found in Experiment 1 can survive a reduction of the presentation time for the face and target from 200 to $100 \mathrm{msec}$, but only if the face stimuli are presented to the left visual field.

\section{Experiment 3}

The RT results are shown in Figure 4. Because there were no significant interactions involving visual field and irrelevant stimuli (all $p \mathrm{~s}>.42$ ), the data from the two visual fields were combined in the subsequent analyses. There was a significant main effect of irrelevant stimuli $[F(2,116)=5.16, p<.01]$. There was also a significant interaction between irrelevant stimuli and cue validity $[F(2,116)=6.50, p<.005]$. As in Experiments 1 and 2, the main effect of irrelevant stimuli was significant in the valid cue condition $[F(2,116)=6.09, p<.005]$. The subsequent pairwise comparisons showed that attractive faces delayed the RTs more than did unattractive faces or the baseline condition $[t \mathrm{~s}(58)=3.96$ and $2.02, p \mathrm{~s}<.0005$ and .05 , respectively]. In the invalid condition, there was a significant main effect of irrelevant stimuli $[F(2,116)=$ $5.80, p<.005]$, where both attractive and unattractive faces delayed the RTs more than did the baseline condition $[t \mathrm{~s}(58)=2.24$ and $3.71, \mathrm{ps}<.03$ and .0005$]$. However, there was no difference between the results for attractive and unattractive conditions ( $p=.37$ ).

Table 1 shows the proportion of trials on which an eye movement was made to the target or irrelevant stimuli following the onset of the central cue. There was no significant main effect of irrelevant stimuli $[F(2,8)=0.18, p=$ $.84]$ or interaction between irrelevant stimuli and cue validity $[F(2,8)=2.52, p=.14]$. The results showed that eye movements occurred infrequently in all conditions, varying between $2 \%$ and $14 \%$ of trials across the 5 participants. The data were collapsed across cue validity, because the overall eye movements to the cued and uncued positions did not differ from each other $[F(1,4)=0.06, p=.82]$. Our results are consistent with Hawkins et al. (1990), who also found no systematic eye movements toward the cued location. The results suggest that the effect of facial attractiveness was not due to foveal fixation on the target or face stimuli, regardless of whether the cue was valid or invalid.

The accuracy results in Experiments 1-3 did not show significant differences between the attractive and unattractive face conditions $(p s>.1)$. The interactions involving irrelevant stimuli and other conditions (cue validity, SOA, and visual field) were also not significant (all $p s>.1$ ).

\section{DISCUSSION}

Our results showed that the presence of attractive faces in these experiments had a detrimental effect on the speed

Table 1

Proportion of Trials on Which an Eye Movement Was Made to the Target or to Irrelevant Stimuli Following the Onset of the Central Cue

\begin{tabular}{ccccccc}
\hline \multirow{2}{*}{ Participant } & Target & $\begin{array}{c}\text { Attractive } \\
\text { Face }\end{array}$ & Target & $\begin{array}{c}\text { Unattractive } \\
\text { Face }\end{array}$ & Target & Baseline \\
\hline 1 & .058 & .072 & .051 & .072 & .038 & .038 \\
2 & .033 & .039 & .044 & .053 & .033 & .042 \\
3 & .020 & .008 & .008 & .014 & .019 & .014 \\
4 & .053 & .049 & .042 & .044 & .069 & .050 \\
5 & .081 & .060 & .063 & .068 & .065 & .072 \\
\hline
\end{tabular}


of judgment for the target orientation. The participants' voluntary allocation of covert attention to the target induced by the central cue was more attenuated by an attractive face than by a less attractive one, even though it was task irrelevant. The speedy detection of facial beauty is consistent with previous findings (Locher, Unger, Sociedade, \& Wahl, 1993; Olson \& Marshuetz, 2005).

When the presentation time for the target and the face image was reduced from $200 \mathrm{msec}$ (Experiment 1) to $100 \mathrm{msec}$ (Experiment 2), the effects of facial attractiveness on spatial attention were found only when the face image was presented to the left visual field. This result suggests a right-hemisphere advantage for processing facial beauty. It may echo the right hemisphere's dominance in processing facial and emotional information (e.g., Kanwisher, McDermott, \& Chun, 1997; Van Strien \& Valstar, 2004). Future imaging studies should help localize the sites in the right hemisphere that have led to the difference between the results of right and left visual field presentations.

In all three experiments, the effect of facial attractiveness was found only when the central cue was valid. When it was invalid, there was no difference between the results in the attractive and unattractive face conditions. This may have been due to the fact that attention in the invalid cue trials already had been directed to the face rather than to the target. Participants had to reorient attention to the target after this. The effect of attractive faces may rely on a shift of spatial attention that was oriented elsewhere. The experiments also revealed that the facial attractiveness effect was primarily associated with the short SOA $(250 \mathrm{msec})$. It is not entirely clear why the same effect was not found in the long SOA $(350 \mathrm{msec})$. It is possible, however, that the participants were better prepared after a long SOA to focus more robustly on the target. Indeed, the overall RTs were faster in this SOA condition.

The attentional bias for attractive faces found in this study signals their biological significance. Researchers have suggested that the preference for attractive faces is deeply rooted in evolution (Langlois et al., 2000; Rhodes, 2006). This Darwinian approach helps to explain why attractive faces could receive more attention. Some researchers have shown that attractive faces carry important information about mate quality (Johnston, 2006). There has been evidence that, although males and females both rate beautiful male and female faces as attractive, their reward circuitry and related brain regions are more strongly activated by faces of the opposite gender (Aharon et al., 2001; Ishai, 2007). However, whether the present finding is modulated by face gender remains to be seen. Due to the limited numbers of trials and of male participants, the present design made it difficult to perform this analysis. This issue will be subject to future investigations.

In summary, our study shows that facial beauty is a powerful stimulus that competes with other visual information for spatial attention. The findings imply that the effect of facial attractiveness extends beyond explicit social behavior and has profound impact right from the entry point of cognitive processing.

\section{AUTHOR NOTE}

This research was supported by a grant from The Royal Society, a Marie Curie Incoming International Fellowship, and a grant from the Natural Science Foundation of China (Project 30700229). We thank David Perrett for offering the face stimuli, Tim Alexander and the reviewers for comments on an earlier version of the manuscript, and Malathy Rengamani and Bryony Hughes for data collection. Address correspondence to J. Sui, Department of Psychology, University of Hull, Cottingham Road, Hull HU6 7RX, England (e-mail: jie.sui@hull.ac.uk).

\section{REFERENCES}

Aharon, I., Etcoff, N., Ariely, D., Chabris, C. F., O’Connor, E., \& Breiter, H. C. (2001). Beautiful faces have variable reward value: fMRI and behavioral evidence. Neuron, 32, 537-551.

Funes, M. J., Lupiáñez, J., \& Milliken, B. (2007). Separate mechanisms recruited by exogenous and endogenous spatial cues: Evidence from a spatial Stroop paradigm. Journal of Experimental Psychology: Human Perception \& Performance, 33, 348-362.

Hawkins, H. L., Hillyard, S. A., Luck, S. J., Mouloua, M., DownING, C. J., \& WoodWARD, D. P. (1990). Visual attention modulates signal detectability. Journal of Experimental Psychology: Human Perception \& Performance, 16, 802-811.

IsHAI, A. (2007). Sex, beauty and the orbitofrontal cortex. International Journal of Psychophysiology, 63, 181-185.

Johnston, V. S. (2006). Mate choice decisions: The role of facial beauty. Trends in Cognitive Sciences, 10, 9-13.

Kanwisher, N., McDermott, J., \& Chun, M. M. (1997). The fusiform face area: A module in human extrastriate cortex specialized for face perception. Journal of Neuroscience, 17, 4302-4311.

Kranz, F., \& IshaI, A. (2006). Face perception is modulated by sexual preference. Current Biology, 16, 63-68.

Langlois, J. H., Kalakanis, L. E., Rubenstein, A. J., Larson, A. D., Hallam, M. J., \& Sмоот, M. T. (2000). Maxims or myths of beauty? A meta-analytic and theoretical review. Psychological Bulletin, 126, 390-423.

Locher, P., Unger, R., Sociedade, P., \& Wahl, J. (1993). At first glance: Accessibility of the physical attractiveness stereotype. Sex Roles, 28, 729-743.

Mäkelä, P., Näsänen, R., Rovamo, J., \& Melmoth, D. (2001). Identification of facial images in peripheral vision. Vision Research, 41, 599-610.

Melmoth, D. R., Kukkonen, H. T., Mäkelä, P. K., \& Rovamo, J. M. (2000). The effect of contrast and size scaling on face perception in foveal and extrafoveal vision. Investigative Ophthalmology \& Visual Science, 41, 2811-2819.

O'Doherty, J., Winston, J., Critchley, H., Perrett, D., Burt, D. M., \& Dolan, R. J. (2003). Beauty in a smile: The role of medial orbitofrontal cortex in facial attractiveness. Neuropsychologia, 41, 147-155.

Olson, I. R., \& Marshuetz, C. (2005). Facial attractiveness is appraised in a glance. Emotion, 5, 498-502.

Palermo, R., \& Rhodes, G. (2007). Are you always on my mind? A review of how face perception and attention interact. Neuropsychologia, 45, 75-92.

RAYNer, K. (1983). Eye movements in reading: Perceptual and language processes. New York: Academic Press.

RHODES, G. (2006). The evolutionary psychology of facial beauty. Annual Review of Psychology, 57, 199-226.

Ro, T., Russell, C., \& Lavie, N. (2001). Changing faces: A detection advantage in the flicker paradigm. Psychological Science, 12, 94-99.

VAn Strien, J. W., \& VAlStar, L. H. (2004). The lateralized emotional Stroop task: Left visual field interference in women. Emotion, 4, 403409.

Winston, J. S., O’Doherty, J., Kilner, J. M., Perrett, D. I., \& Dolan, R. J. (2007). Brain systems for assessing facial attractiveness. Neuropsychologia, 45, 195-206.

(Manuscript received January 2, 2008; revision accepted for publication September 17, 2008.) 